\title{
THE THIRTIETH SUMMER MEETING OF THE AMERICAN MATHEMATICAL SOCIETY
}

The thirtieth summer meeting of the Society was held at Vassar College, Poughkeepsie, New York, on Thursday and Friday, September 6-7, 1923, the college opening its buildings for the entertainment of the visitors and thus contributing greatly to the success of the sessions. The Mathematical Association of America held its meetings immediately preceding and a joint session of the two societies was arranged for Thursday afternoon, at which addresses were delivered by Professor L. J. Mordell, of the University of Manchester, and Professor R. C. Archibald, of Brown University. At the joint dinner on Thursday evening, President McCracken of Vassar College welcomed the visiting mathematicians and spoke on the relation of the undergraduate college to research. Tea was served every afternoon after the sessions at the home of Professor and Mrs. H. S. White. At the last session, it was voted to express to Vassar College and to the department of mathematics the thanks of the Society for their generous hospitality and for the admirable arrangements.

The attendance included the following seventy-nine members of the Society:

Agard, Archibald, G. N. Armstrong, Babb, Bacon, Barney, E. R. Beckwith, S. R. Benedict, Bernstein, E. W. Brown, H. S. Brown, J. A. Bullard, W. G. Bullard, R. W. Burgess, Cairns, G. M. Conwell, Copeland, Court, L. D. Cummings, Dimick, Dines, Eiesland, Feldstein, W. B. Ford, Glenn, M. C. Graustein, W. C. Graustein, Grove, E. R. Hedrick, A. M. Howe, Huntington, Ingels, Kazarinoff, Kline, W. D. Lambert, F. P. Lewis, Luck, McCain, McDonnell, MacDuffee, Mathews, H. H. Mitchell, C. N. Moore, Mordell, Morenus, C. C. Morris, Nassau, Pierpont, Pitcher, Post, Rambo, Ranum, Reddick, I. J. Reed, R. G. D. Richardson, E. D. Roe, J. R. Roe, Rosenbach, Seely, Sinclair, Slaught, Slobin, C. E. Smith, D. E. Smith, Gertrude Smith, W. M. Smith, M. J. Sperry, B. M. Turner, Tyler, Vandiver, Veblen, M. E. Wells, H. S. White, Whittemore, Constance Wiener, Norbert Wiener, E. W. Wilson, Worthington, J. W. Young.

At the meeting of the Council, the following twenty-seven persons were elected to membership in the Society: 
Professor Samuel Latimer Boothroyd, Cornell University;

Mr. Leonidas Hamlin Bunyan, University of Wisconsin;

Miss May Bryan Carter, Western College;

Professor Jacques Chapelon, University of Lille;

Rev. Gabriel Hippolytus Collignon, Loyola University;

Mr. Paul Sumner Dwyer, Pennsylvania State College;

Miss Lois Wilfred Griffiths, University of Washington;

Professor Lilian May Hackney, Marshall College;

Professor George Wellman Hess, Whitworth College;

Mr. Arthur Owen Hickson, Brown University;

Captain Grafton S. Kennedy, Ordnance Department, United States Army;

Mr. Harry Raymond Kimball, San Francisco, Calif.;

Mr. Harry Levy, Princeton University;

Dr. Elsie Jeannette McFarland, University of California;

Mr. Robert Houghton Marquis, Pennsylvania State College;

Professor Rhesa Lancaster Newlin, Guilford College;

Professor Niels Erik Nörlund, University of Copenhagen;

Mr. Howard Percy Robertson, University of Washington;

Mr. Henry Allen Robinson, Johns Hopkins University;

Professor Lao Genevra Simons, Hunter College;

Mr. Marshall Harvey Stone, Harvard University;

Mr. John Clement Tinner, Chicago, Ill.;

Lieutenant Lawrence Wainwright, United States Navy;

Professor Benjamin Lewis Waits, Alcorn College;

Professor Anne Marie Whelan, Olivet College;

Miss Evelyn Prescott Wiggin, Brown University;

Mr. Hugh Herbert Wolfenden, Grimsby, Ontario.

Forty-nine applications for membership were received. The Secretary announced that the following members of the London Mathematical Society had accepted membership under the reciprocity agreement since the April meeting.

Mr. Charles G. F. James, London;

Professor Louis Joel Mordell, University of Manchester.

The Council announced the appointment of the following committees: Professors Tyler, E. R. Hedrick, R. G. D. Richardson, Slaught, Veblen, H. S. White and J. W. Young on revision of the by-laws; Professors Eisenhart, Birkhoff and Dickson on nomination of officers; Professors Veblen, Birkhoff, Eisenhart, E. R. Hedrick, Slaught and J. W. Young on printing of the journals. Professor Birkhoff represented the Society at the inauguration of President Stratton of the Massachusetts Institute of Technology on June 11, 1923.

It was announced that the Guarantee Fund for the expenses 
of the endowment campaign had been raised and that the campaign itself would be formally opened in October. The whole-hearted cooperation of the members of the Society was invited. The committee on incorporation reported that it was confidently expected that the necessary legal forms could be attended to so that the incorporated body would supersede the unincorporated before the end of the year.

A resolution was adopted sanctioning the establishment of a lectureship to be known as the Josiah Willard Gibbs Lectureship, the lecture to deal in semi-popular form with some aspect of mathematics or its applications. The President was authorized to appoint a committee to make arrangements for the first lecture, which will probably be given in New York City during the winter of 1923-24.

In view of the anticipated meeting of the International Mathematical Congress in Canada in the summer of 1924 the Society decided to omit its summer meeting for that year.

At the meeting of the Society and the Association it was voted to request the Secretaries of the two organizations to send a letter to the Physico-Mathematical Society of Japan, expressing the sympathy of American mathematicians for their colleagues in Japan under the calamity that has befallen their country through the great earthquake.

At the joint session the following papers were read:

I. An introductory account of the arithmetical theory of algebraic numbers and its recent development, by Professor L. J. Mordell. (Address delivered at the request of the American Mathematical Society.)

II. Mathematicians and music, by Professor R. C. Archibald. (Address of the retiring President of the Mathematical Association of America.)

President Oswald Veblen presided at the regular sessions of the Society, relieved by Professor H. S. White. Titles and abstracts of the papers read at these sessions follow below. The papers of Professor R. L. Moore, Dr. Murray, Professor Altshiller-Court, Professor Murnaghan, Dr. Walsh, Mr. Thomas, Mr. McDonnell, Mr. Michal, and Professor Evans 
were read by title; Mr. Rainich was introduced by Professor Kasner, and Mr. Michal by Professor Evans.

1. Professor H. S. White: Note on five points and a cyclic correspondence.

Five given numbers in a given cyclic order determine completely a $(2,2)$ correspondence. The explicit equation is deduced, and an algebraic proof of its periodicity.

2. Professor B. A. Bernstein: A generalization of the syllogism.

This paper will appear in full in an early issue of this BuLLETIN.

3. Professor B. A. Bernstein: Operations with respect to which the elements of a boolean algebra form a group.

Let $a^{\prime}$ denote the negative of a boolean element $a, a+b$ the logical sum of $a$ and $b$, and $a b$ their logical product; the author pointed out in a previous paper that $a b^{\prime}+a^{\prime} b$ and $a b+a^{\prime} b^{\prime}$ are operations with respect to each of which the elements of a boolean algebra form an abelian group. In this paper he determines all the operations of a boolean algebra with respect to which the elements form a group in general and an abelian group in particular.

4. Mr. H. S. Vandiver: A new type of criteria for the first case of Fermat's last theorem.

By transforming the criteria of Kummer, the author shows that if $x^{p}+y^{p}+z^{p}=0$ is satisfied in integers $x, y$, and $z$, not zero and prime to the odd prime $p$, then

$$
1+\frac{1}{2^{2}}+\frac{1}{3^{2}}+\cdots+\frac{1}{u^{2}} \equiv 0 \quad(\bmod p),
$$

where $u$ is the greatest integer in $p / 3$. The paper will be offered for publication to the AnNals of Mathematics.

5. Mr. H. S. Vandiver: A method for finding a factor of an integer of the form $8 n+1$.

If an integer $m$ of the form $8 n+1$ is the product of two prime factors, then each factor has the same residue modulo 8. The author shows that $m$ may be represented in at least two different ways in one of the forms $x^{2}+y^{2}, x^{2}+2 y^{2}$, $2 x^{2}-y^{2}$, where $x<\sqrt{p}, y<\sqrt{p}$. The factors of $m$ are found from the different representations. The paper will be offered for publication to this BULLETIN. 
6. Mr. G. Y. Rainich: The distribution of primes and the finiteness of the number of discriminants with a given number of classes.

In this paper a proof of the following proposition is given: the number of integers $m$ for which the ratio of the whole number of primes between $m$ and $m^{2}$ to the number of primes of the form $x^{2}+x y+m y^{2}$ between the same limits does not exceed a given value is finite. It seems probable that when the number of classes of the discriminant $1-4 \mathrm{~m}$ is fixed the aforesaid ratio does not exceed a certain value (which is very near to the double of the number of classes); should this be proved, the finiteness of the number of discriminants with a given number of classes would be established. The degree of non-unicity of decomposition in prime factors is characterized by the maximum number of factors in a product which is divisible by a prime without any partial product being divisible by a prime; the connection of this constant in the case of quadratic fields with the structure of the group of classes is shown. It is proved that when the number of classes is unity all the numbers $x^{2}$ $+x y+m y^{2}$ below $m^{2}$ are primes (for $x$ and $y$ relative primes).

7. Professor O. E. Glenn: A complete system of differential parameters of orders $<3$ of the binary differential cubic.

This paper, combined with two communicated previously as preliminary reports (this Bulletin, April and May, 1923), forms a memoir entitled $0 n$ the reduction of differential parameters in terms of finite sets, with remarks concerning differential invariants of analytic transformations, which has been offered to the American Journal of Mathematics. The finite system determined for the cubic belongs to the domain of rational polynomials in derivatives of orders $\leqq 3$ of the functions $x_{i}=\varphi_{i}\left(y_{1}, y_{2}\right)(i=1,2)$ of the transformations.

8. Professors E. R. Hedrick and Louis Ingold: Analytic and non-analytic functions in three dimensions.

In a paper by Hedrick, Ingold, and Westfall, soon to appear in the Journal De Mathématiques, it is shown that analytic functions of a complex variable may be distinguished from nonanalytic functions by the fact that the Tissot indicatrix becomes a circle in the analytic case; also by the fact that certain characteristic directions become indeterminate. In the present paper the authors show that for transformations of space into itself, there is an ellipsoid at each point which plays a rôle 
analogous to that of the Tissot indicatrix in the plane, and there are at each point characteristic directions. In particular they study those transformations for which this ellipsoid reduces to a sphere, and the characteristic directions become indeterminate. It is found that these transformations are analogous to analytic functions. The transformations coincide with conformal transformations of space, and the set of functions defining them satisfy equations analogous to the Cauchy-Riemann equations; also each of these functions satisfies an equation analogous to Laplace's equation.

9. Professor R. L. Moore: A connected and regular point set which contains no arc.

It is shown that there exists a connected point set which is regular (connected im kleinen) at every one of its points but which contains no simple continuous arc.

10. Professor R. L. Moore: Concerning the sum of a countable infinity of continua in the plane.

Sierpinski has shown that there exists no bounded plane continuum which is the sum of a countable infinity of mutually exclusive closed point sets. He has raised the question whether this result would still hold good if the word "bounded" were omitted. In the present paper it is shown that, for the special case where the closed point sets in question are all continua, this question may be answered in the affirmative.

11. Professor R. L. Moore: A continuum considered as the sum of its prime elements.

Hans Hahn has recently introduced the notion of the prime elements (Primteile) of a continuum and has proved a theorem which may be interpreted to mean that, with respect to its prime elements considered as points, every continuum which is irreducible between two points is a simple continuous arc which has those points as end points. In the present paper it is shown, among other things, that every bounded continuum whatsoever is a continuous curve with respect to its prime elements considered as points.

12. Professor Mary E. Sinclair: The brachistochrone with variable end points.

Conditions for fixed end points require that the extremals be single arches of inverted cycloids. The first derivatives of the extremal-integral, $J(u, v)$, where $u$ and $v$ are the parameters of two fixed plane curves on which the end points vary, 
gives the known transversality conditions. Its second derivatives give conditions involving the curvatures of the fixed curves and the parameters of the cycloid at the end points, such that if but one end point vary, its focal point on the arch may be studied. If both end points vary, the second derivatives give inequalities which may be interpreted as restricting the curvature of one of the fixed curves when that of the other and the parameters of the cycloid are given. A geometric interpretation of these results is given. Sufficient conditions for a relative minimum follow from the theorem of Hahn used by Merrill in his generalization of the problem of Dido.

13. Professor R. G. D. Richardson: A new necessary condition for relative extrema in quadratic and hermitian forms.

If $A=\sum_{i j}^{1, n} a_{i j} x_{j} x_{i}, B=\sum_{i j}^{1, n} b_{i j} x_{j} x_{i}$ be real quadratic forms of which $B$ is positive definite, the minimum of $A$ subject to the condition $B=1$ must be one of the $n$ solutions $x_{1}{ }^{(i)}, \cdots, x_{n}{ }^{(i)}$ of the equations $\sum_{j}\left(a_{i j}-\lambda b_{i j}\right) x_{j}=0$, corresponding to one of the $n$ zeros $\lambda_{i}$ of the determinant $\left|a_{i j}-\lambda b_{i j}\right|$. The new necessary condition for a minimum obtained by the author determines the particular solution furnishing the minimum. For the $\lambda_{i}$ which is desired, it is necessary that in the determinant $\left|a_{i j}-\lambda_{i} b_{i j}\right|$ the sequence $\sigma_{i}(i=0, \cdots, n)$ of sums of all the principal minors obtained by deleting $i$ rows and the same $i$ columns show no permanence of sign. This condition together with those already known forms a sufficient set.

More generally, when the linear conditions $\sum_{i j} b_{i j} x_{j}{ }^{(1)} x_{i}$ $=0, \cdots, \sum_{i j} b_{i j} x_{j}{ }^{(m)} x_{i}=0$ are added to the problem, the minimum is again a solution of the same linear equations and a similar new necessary condition on minors of the bordered determinant completes the set of sufficient conditions.

The same theory applies also in the case of hermitian forms.

14. Professor C. N. Moore: On the summation of trigonometric series by Euler's method.

In this paper it is shown that while Euler's method of summation will serve to sum certain divergent trigonometric series, it is less effective than Cesàro's method when applied to Fourier's series. For it follows from a general criterion due to Lebesgue that Euler's method will not serve to sum at points of continuity of the function developed all the Fourier's series corresponding to functions having a Lebesgue integral, whereas it is a well known theorem that Cesàro's method has this property. 
15. Professor L. L. Dines: Concerning a suggested and discarded generalization of the Weierstrass factorization theorem.

This paper will appear in full in an early issue of this BuLLETIN.

16. Professor L. L. Dines: A theorem on the factorization of polynomials of a certain type.

Polynomials of the form $P \equiv y^{n}+P_{n-1}(x) y^{n-1}+\cdots+$ $P_{1}(x) y+P_{0}(x)$, where the coefficients $P_{j}(x)$ are power series converging in a sufficiently restricted neighborhood of the origin and vanishing with $x$, are of importance in the theory of implicit functions. In the present paper, the author gives a direct algebraic proof of the following algebraic theorem relative to such polynomials: If the characteristic line (Newton polygon) of the polynomial $P$ is a broken line of $k$ segments, then $P$ is a product of $k$ polynomials of the same type as $P$, each of which has an unbroken characteristic line the same in length and direction as one segment of the characteristic line of $P$. The theorem is for this type of polynomials a converse of the "Theorem on the Product" proved by Blumberg (TransacTIONS OF THIS SocIETY, vol. 17, p. 530) for a very general class of polynomials defined postulationally.

17. Mr. Donat Kazarinoff: The scientific work of $A$. $M$. Liapounoff.

This paper gives an account of the scientific work of the eminent Russian mathematician A. M. Liapounoff (born in Yaroslavl, 1857; died in Odessa, 1918). His achievements won wide recognition; he was a member of the Russian Academy of Sciences, a foreign member of the Accademia dei Lincei, a correspondent of the Académie des Sciences, etc. His basic contributions are on the figures of equilibrium of a homogeneous rotating liquid.

The present paper depends for its information almost entirely upon the obituary memoir by V. A. Stekloff, Bulletin of the Russian Academy of Sciences, ser. 6, vol. 13 (1919), pp. 367-388.

18. Dr. Norbert Wiener: The quadratic variation of a function.

In this paper, a discussion of the second degree analog of the total variation of a function is employed to establish certain inequalities connecting the total variation of a function, its saltus, and its Fourier coefficients. 
19. Dr. F. H. Murray: Certain orbits with arbitrary masses in the problem of three bodies.

This paper is devoted chiefly to the study of certain special cases of the problem of three bodies, by methods in which the notion of "system of invariant relations" as defined by Poincaré plays an important rôle. After certain properties of differential systems which admit a set of invariant relations have been developed, some qualitative results are obtained concerning isosceles triangle solutions with axis of symmetry. The equations of the problem of three bodies in the plane are given in a comparatively simple form, with the aid of which solutions asymptotic to the straight line and equilateral triangle solutions, respectively, are obtained. These results complete, on certain points, the researches of D. Buchanan concerning these solutions.

20. Professor W. C. Graustein: Applicability with preservation of both curvatures.

This paper will appear in full in an early issue of this BuLLETIN.

21. Professor W. C. Graustein: Isometric W-surfaces.

This paper contains a complete classification of isometric $W$-surfaces and a discussion of the new types found. Special attention is given to isometric $W$-surfaces admitting continuous deformations into themselves.

22. Mr. G. Y. Rainich: A new kind of representation of curved space.

Given a surface $S$ and a plane $P$ we draw through a point $A$ of $S$ the normal to $S$ and the perpendicular to $P$. We call the intersection of the bisector of the angle formed by these two lines with $P$ the generalized stereographic projection of $A$. Vectors belonging to $S$ (cf. an article by the author in the Proceedings of the National Academy, June, 1923) can also be projected stereographically, and from this arises a new kind of representation of tensors belonging to the surface through tensors of a plane, which has an advantage over the usual representation so far as algebraic relations are concerned, since no such distinctions as that between covariant and contravariant quantities are introduced. Differentiation, on the other hand, is more complicated in this system. Projecting the neighborhood of a point stereographically on the tangent plane at this point, and using cartesian coordinates in this 
plane, we obtain "semi-cartesian" coordinates for the representation of the surface. We can use a similar representation for higher spaces and also for curves on a plane.

23. Professor Nathan Altshiller-Court: On two circles.

The pair of lines joining the two points of intersection of two circles to a variable point on one of these circles determine in the second circle a chord of constant length. This length $x$ is given by the proportion $x: s=d: p$, where $s, d, p$ are the lengths of the common chord, the line of centers, and the radius of the first circle respectively. The author applies this result to a coaxial system of circles, to various circles connected with the triangle, etc., and gives a projective generalization of the proposition.

24. Professor F. D. Murnaghan: A minimum problem in elementary geometry.

The problem of finding the point the sum of whose distances to four points in space is a minimum was proposed by Steiner and has been considered by many geometers, notably by R. Sturm. Although Steiner gave a hint of the proper mode of attack, his hint seems to have escaped attention. It is apparent that if planes each through one of the four points perpendicular to its join to a fifth point form an equifacial tetrahedron, this fifth point will make $r_{1} \pm r_{2} \pm r_{3} \pm r_{4}$ a minimum or a maximum. To find such a fifth point $x$ (using as coordinates perpendiculars on the faces of the tetrahedron formed by the four given points) note that the pedal tetrahedron of its isogonal conjugate $y=1 / x$ will also be equifacial, and hence have its opposite edges equal. There are therefore 8 points $y$, the base points of the net of quadrics $y_{1}{ }^{2}+y_{2}{ }^{2}+2 c_{12} y_{1} y_{2}=y_{3}{ }^{2}+y_{4}{ }^{2}+2 c_{34} y_{3} y_{4}, y_{1}{ }^{2}+y_{3}{ }^{2}+2 c_{13} y_{1} y_{3}$ $=y_{2}{ }^{2}+y_{4}{ }^{2}+2 c_{24} y_{2} y_{4}, y_{1}{ }^{2}+y_{4}{ }^{2}+2 c_{14} y_{1} y_{4}=y_{2}{ }^{2}+y_{3}{ }^{2}$ $+2 c_{23} y_{2} y_{3}$, where $c_{r s}$ is the cosine of the internal angle between the faces $r$ and $s$ of the tetrahedron of reference.

25. Dr. J. L. Walsh: A generalization of evolutes.

Let points $P_{1}$ and $P_{2}$ trace two curves $C_{1}$ and $C_{2}$ so that the tangents to $C_{1}$ and $C_{2}$ at $P_{1}$ and $P_{2}$ are parallel. Let the point $P$ be determined so that it divides the segment $P_{1} P_{2}$ in the constant ratio $m_{1}: m_{2} ; P$ traces a curve $C$. Then the centers of curvature $Q_{1}, Q_{2}, Q$ of $C_{1}, C_{2}, C$ at corresponding points $P_{1}, P_{2}$, $P$ are such that $Q$ divides the segment $Q_{1} Q_{2}$ in the ratio $m_{1}: m_{2}$. The circles of curvature of $C_{1}, C_{2}, C$ at $P_{1}, P_{2}, P$ have as common center of similitude the point of tangency of the variable 
line $P_{1} P_{2}$ with its envelope. Consider the family of curves found by varying the ratio $m_{1}: m_{2}$. An infinity of curves of this family can be traced by points of an elastic string which moves so as to wrap itself on the envelope of the line $P_{1} P_{2}$.

26. Mr. J. M. Thomas: Congruences of circles studied with reference to the surface of centers.

The surface of centers $S$, rather than the envelope of the planes, is used as the framework of reference. Certain theorems relating to $S$ in general are first proved. The main part of the paper is devoted to congruences $C$ characterized as follows: each point $P$ of a circle of the congruence is on a surface whose tangent plane at $P$ contains the radius to $P$ and cuts the plane of the circle at an angle $90^{\circ}-\varphi$, where $\varphi$ is a function at most of the two parameters of the circle. Moreover, for a congruence $C$ the surface $S$ is distinct from the envelope of the planes. When all the circles have the same radius and $\varphi$ is constant, it is found that the planes of the circles are the osculating planes of a single parameter family of curves of constant torsion on $S$. The case where $S$ degenerates into a curve is considered. For a cyclic system the curve of centers must be plane unless the circles are in the normal planes to the curve.

27. Professor John Eiesland: A theorem in relativity.

The following theorem is proved: Necessary and sufficient conditions that a centro-symmetric space with line element $-d s^{2}=\varphi_{2} d r^{2}+\varphi_{3}\left(d \theta^{2}+\sin ^{2} \theta d \varphi^{2}\right)-\varphi_{1} d t^{2}, \varphi_{1}, \varphi_{2}$ and $\varphi_{3}$ being arbitrary functions of $r$ and $t$, shall be reducible to the static form are $\varphi_{1} \varphi_{2} G_{1}{ }^{0}=\left(\partial \varphi_{3} / d r\right)\left(\partial \varphi_{3} / d t\right)$, and

$$
\varphi_{1} \varphi_{2} \varphi_{3}\left(G_{1}-G_{0}\right)=-\psi^{\prime}\left[\varphi_{1}\left(\frac{d \varphi_{3}}{d r}\right)^{2}+\varphi_{3}\left(\frac{d \varphi_{3}}{d t}\right)\right],
$$

where $\psi$ is an arbitrary function of $\varphi_{3}$. It follows that the Einstein solar field is necessarily static, ${ }^{*}$ and that the same is true of other spaces of importance in relativity theory.

28. Professor John Eiesland: On a generalization of Kummer's surface in odd n-space.

Adopting as space element the $\infty^{n}$ flats $\sigma_{0} x_{i}-\sigma_{n / 2} y_{i}+\rho_{i}$ $=0, \sigma_{0} z+\sum \sigma_{i} y_{i}+\rho_{n / 2}=0, \sigma_{n / 2} z+\sum \sigma_{i} x_{i}-\rho_{0}=0$ in

* For an analytic proof of this proposition based on the existence-theorem for a pair of differential equations, see Birkhoff, Relativity and Modern Physics, Cambridge, 1923, pp. 253-256. 
$S_{n-1}$, where $\sum \rho_{i} \sigma_{i}=0$, the author considers the quadratic flat-complex $\varphi=\sum a_{i}\left(\rho_{i}{ }^{2}+\sigma_{i}{ }^{2}\right)+2 \sum b_{i} \rho_{i} \sigma_{i}=0$, and the singular surface of this complex. This surface is of the eighth order and eighth class, it is self dual, and has a 2-spread for locus of double-spreads. If Klein's coordinates $y_{2 i+1}=\rho_{i}$ $+\sigma_{i}, i y_{2 n+2}=\rho_{i}-\sigma_{i}$ are introduced, the complex takes the form $\sum_{1}^{n+1} y_{i}{ }^{2}=0, \sum_{1}^{n+1} k_{i} y_{i}{ }^{2}=0$. The coordinates of the flats $y_{i}$ may be represented as hyperelliptic functions of $n$ parameters $\lambda_{i}$, and therefore also the coordinates of the singular surface may be similarly represented.

29. Mr. John McDonnell: A note on chapter 2 of volume 3 of L. E. Dickson's History of the Theory of Numbers.

In the above-mentioned chapter various theorems are stated concerning the expressibility of $4 p$ in the form $a^{2}+27 b^{2}$ when $p$ is a prime $=6 n+1$. In the present paper it is proved that $p$ itself is expressible in the form $a^{2}+27 b^{2}$ if $2^{(p-1) / 3}$ $\equiv 1(\bmod p)$ and conversely.

30. Mr. A. D. Michal: Integro-differential invariants of oneparameter groups of Volterra transformations.

The author continues the discussion of integro-differential invariants, in particular functionals of the form $f\left[\begin{array}{ll}1 & 1 \\ y & y^{\prime} \\ y^{\prime}\end{array}\right]$ which are "analytic," and shows that a necessary and sufficient condition that such a functional be invariant of a one-parameter group of linear functional transformations of Volterra type is that it satisfy a certain completely integrable equation in partial functional derivatives. The expansion of the solution of this equation is obtained.

31. Professor G. C. Evans: The dynamics of monopoly.

The author considers a problem in the dynamics of economics, namely, the change from one price to another under conditions of monopoly, when the demand function involves the rate of change of price as well as the price itself. The total profit over the stretch of time involved in the change shall be a maximum. The demand function is taken as of the first degree in the price and the rate of change of the price, and the cost is assumed to be a quadratic function of the amount produced.

R. G. D. Richardson, Secretary. 